OUTP-97-23P

$\mathrm{DSF} / 24-97$

hep-th/9706107

July 24, 2021

\title{
Target Space Duality in Noncommutative Geometry
}

\author{
FEDELE LIzZ用 AND Richard J. SzaBoil \\ Department of Physics - Theoretical Physics \\ University of Oxford \\ 1 Keble Road, Oxford OX1 3NP, U.K.
}

\begin{abstract}
The structure of spacetime duality and discrete worldsheet symmetries of compactified string theory is examined within the framework of noncommutative geometry. The full noncommutative string spacetime is constructed using the Fröhlich-Gawędzki spectral triple which incorporates the vertex operator algebra of the string theory. The duality group appears naturally as a subgroup of the automorphism group of the vertex operator algebra and spacetime duality is shown to arise as the possibility of associating two independent Dirac operators, arising from the chiral structure of the worldsheet theory, to the noncommutative geometry.
\end{abstract}

PACS: 02.40.+m, 04.60.+n, 11.17.+y

${ }^{*}$ Permanent Address: Dipartimento di Scienze Fisiche, Università di Napoli Federico II and INFN, Sezione di Napoli, Italy.

${ }^{\dagger}$ Work supported in part by the Natural Sciences and Engineering Research Council of Canada. 
One of the key tools to the understanding of the spacetime structure of string theory is the concept of 'duality' (see [1] for a review). A duality in string theory relates a geometry of the target space in which the strings live to an inequivalent one. The mapping between distinct geometries is a symmetry of the quantum string theory. The equivalence between such geometries from the string theoretic point of view leads to the notion of a stringy or quantum spacetime which forms the moduli space of string vacua and describes the appropriate stringy modification of classical general relativity. Dualities have been exploited recently to relate apparently distinct string theories. A present model for the spacetime structure of superstring theory is 'M Theory' [2] which subsumes all five consistent superstring theories in ten dimensions via duality transformations and contains 11-dimensional supergravity as its low-energy limit.

T-duality, which relates large and small radius circles, leads to a fundamental length scale in string theory, determined by the Planck length $l_{P}$. A common idea is that at distances smaller than $l_{P}$ the conventional notion of a spacetime geometry is inadequate to describe its structure. The string configurations are conjectured to be smeared out and the notion of a 'point' in the spacetime ceases to make sense. A recent candidate theory for this picture is the effective matrix field theory for D-branes [3] in which the spacetime coordinates are described by noncommuting matrices. In this Letter we shall discuss how the effective string spacetime and its associated dualities can be described using the techniques of noncommutative geometry [4]. These mathematical tools are particularly well-suited to the study of the structure of the stringy spacetime, in that it views it not as a set of coordinates, but rather in terms of the set of fields defined on it.

The basic object which describes a metric space in noncommutative geometry is the spectral triple $\mathcal{T}=(\mathcal{H}, \mathcal{A}, D)$, where $\mathcal{H}$ is a Hilbert space, $\mathcal{A}$ is a $C^{*}$-algebra of bounded operators acting on $\mathcal{H}$, and $D$ is a Dirac operator on $\mathcal{H}$. A spin-manifold $M$ with metric $g_{\mu \nu}$ is described by choosing $\mathcal{H}=L^{2}(\operatorname{spin}(M))$, the square-integrable spinors on $M$, and the abelian algebra $\mathcal{A}=C^{0}(M)$ of continuous complex-valued functions on $M$ acting by pointwise multiplication in $\mathcal{H}$. This is the canonical $C^{*}$-algebra associated with any manifold, and it determines the topology of a space through the continuity criterion. In fact, there is a one-to-one correspondence between the set of all topological spaces and the collection of commutative $C^{*}$-algebras, and therefore the study of the properties of spacetime manifolds can be substituted by a study of the properties of abelian $C^{*}$-algebras. The usual Dirac operator $D=i g^{\mu \nu} \gamma_{\mu} \nabla_{\nu}$ then describes the Riemannian geometry of the manifold, where the real-valued gamma-matrices obey the Clifford algebra $\left\{\gamma_{\mu}, \gamma_{\nu}\right\}=2 g_{\mu \nu}$ and $\nabla_{\mu}$ is the usual covariant derivative constructed from the spin-connection. Thus, roughly speaking, $D$ is the "inverse" of the infinitesimal $d x$ which determines geodesic distances in the spacetime.

A quantum theory of point particles on $M$ naturally supplies $\mathcal{H}=L^{2}(\operatorname{spin}(M))$ and can therefore be thought of as describing an ordinary spacetime $M$. In the case of string 
theory then, we can anticipate that the full stringy spacetime is described by a noncommutative geometry, i.e. a spectral triple with a noncommutative algebra $\mathcal{A}$. In this case the notion of a 'space' breaks down, as it is in general impossible to speak of points. In the low-energy limit of the string theory, the vibrational modes are negligible and the model describes ordinary point-like particles corresponding to an ordinary (commutative) spacetime. Thus in the low-energy limit of the string theory, $\mathcal{T}$ should contain a subspace $\mathcal{T}_{0}=\left(L^{2}(\operatorname{spin}(M)), C^{0}(M), i g^{\mu \nu} \gamma_{\mu} \nabla_{\nu}\right)$ representing an ordinary spacetime manifold $M$ at large distance scales.

The symmetries of the quantum spacetime $\mathcal{T}$ then determine the stringy effects on the geometry of spacetime $\mathcal{T}_{0}$ represented by ordinary quantum field theory. In the following we shall construct the spectral triple $\mathcal{T}$ associated with the stringy geometry and show that the full duality group of spacetime is determined in a very simple fashion by the group of automorphisms of the appropriate algebra $\mathcal{A}$. The duality symmetries of the spacetime emerge as the possibility of assigning two independent Dirac operators to the spectral triple $\mathcal{T}$ associated with strings, so that duality is simply a consequence of a change of metric representing a redefinition of distances in the string spacetime. These automorphisms leave the spectral triple $\mathcal{T}$ invariant and correspond to internal symmetries of the quantum geometry. We shall also construct the low-energy projections $\mathcal{T}_{0}$ and illustrate how the duality isomorphisms of $\mathcal{T}$ correspond to the more familiar notions of target space duality in string theory. These projections then illuminate the full structure of the stringy modification of classical general relativity.

The effective low-energy spectral triple has been constructed in the context of D-brane field theory recently in [5]. Here we shall follow the proposal of Fröhlich and Gawędzki [6] that the noncommutative geometry of the string spacetime is provided by choosing for $\mathcal{A}$ the vertex operator algebra of the underlying conformal field theory for the strings. For illustration we shall study the simplest situation with a flat $d$-dimensional target space of bosonic strings compactified to a torus $T^{d}=\left(S^{1}\right)^{d} \cong \mathbb{R}^{d} / 2 \pi \Gamma$, where $\Gamma$ is a lattice of rank $d$ with metric $g_{\mu \nu}$ of Euclidean signature and with inverse $g^{\mu \nu}$. The classical embedding functions of the strings in such a target space are the chiral multivalued Fubini-Veneziano fields (in units with $l_{P}=1$ )

$$
X_{ \pm}^{\mu}\left(z_{ \pm}\right)=x_{ \pm}^{\mu}+i g^{\mu \nu} p_{\nu}^{ \pm} \log z_{ \pm}+\sum_{k \neq 0} \frac{1}{i k} \alpha_{k}^{( \pm) \mu} z_{ \pm}^{-k}
$$

where $\mu=1, \ldots, d$ and $\left(\alpha_{k}^{( \pm) \mu}\right)^{*}=\alpha_{-k}^{( \pm) \mu}$. Here we take the strings to be closed with worldsheet the cylinder $(\tau, \sigma) \in \mathbb{R} \times S^{1}$ and $z_{ \pm}=\mathrm{e}^{-i(\tau \pm \sigma)}$. The constant modes $x_{ \pm}^{\mu}$ describe the center of mass coordinates of the string and $p_{\mu}^{ \pm}$the corresponding momenta. Singlevaluedness restricts these left-right momenta to $p_{\mu}^{ \pm}=\frac{1}{\sqrt{2}}\left(p_{\mu} \pm d_{\mu \nu}^{ \pm} w^{\nu}\right)$ where $\left\{w^{\mu}\right\} \in \Gamma$ are the winding numbers representing the number of times that the multivalued string functions wrap $\sigma$ around the circles of the torus $\left(S^{1}\right)^{d}$, and $\left\{p_{\mu}\right\} \in \Gamma^{*}$ are the momenta of the winding modes in the target space, with $\Gamma^{*}$ the lattice dual to $\Gamma$. The 'background' matrices 
$d_{\mu \nu}^{ \pm}=g_{\mu \nu} \pm \beta_{\mu \nu}$ are constructed from the spacetime metric $g_{\mu \nu}$ and an antisymmetric instanton tensor $\beta_{\mu \nu}$ which does not contribute to the classical dynamics of the strings. Upon quantization the zero modes $x_{ \pm}^{\mu}, p_{\mu}^{ \pm}$become a canonically conjugate pair and the $\alpha$ 's (oscillatory modes) act as creation and annihilation operators on a commuting pair of Fock spaces $\mathcal{F}^{ \pm}$built on vacuum states $|0\rangle_{ \pm}$. The Hilbert space of the string theory is thus $\mathcal{H}_{X}=L^{2}\left(T^{d}\right)^{\Gamma} \otimes \mathcal{F}^{+} \otimes \mathcal{F}^{-}$where $L^{2}\left(T^{d}\right)^{\Gamma}=\bigoplus_{\left\{w^{\mu}\right\} \in \Gamma} L^{2}\left(T^{d}\right)$ are $L^{2}$-spaces labelled by $w^{\mu}$.

We shall take the Hilbert space $\mathcal{H}_{X}$ as the first input into the construction of the string spacetime $\mathcal{T}$. The next ingredient is an algebra $\mathcal{A}_{X}$ acting on $\mathcal{H}_{X}$ which represents its topology. For this we exploit the operator-state correspondence which relates the Hilbert space $\mathcal{H}_{X}$ to an appropriate operator algebra. We define [6] $\mathcal{A}_{X}$ to be the algebra generated by the smeared vertex operators

$$
V_{\Omega}\left(q^{+}, q^{-}\right)=\int \frac{d z_{+} d z_{-}}{4 \pi z_{+} z_{-}} \mathcal{V}_{q^{+} q^{-}}^{\Omega}\left(z_{+}, z_{-}\right)
$$

where $q_{\mu}^{ \pm}=\frac{1}{\sqrt{2}}\left(q_{\mu} \pm d_{\mu \nu}^{ \pm} v^{\nu}\right)$, the local vertex operators are

$$
\begin{gathered}
\mathcal{V}_{q^{+} q^{-}}^{\Omega}\left(z_{+}, z_{-}\right)=: i V_{q^{+} q^{-}}\left(z_{+}, z_{-}\right) \\
\times \prod_{j} \frac{r_{\mu}^{(+) j}}{\left(n_{j}-1\right) !} \partial_{z_{+}}^{n_{j}} X_{+}^{\mu} \prod_{k} \frac{r_{\nu}^{(-) k}}{\left(m_{k}-1\right) !} \partial_{z_{-}}^{m_{k}} X_{-}^{\nu}:
\end{gathered}
$$

and $V_{q^{+} q^{-}}\left(z_{+}, z_{-}\right)=(-1)^{q_{\mu} w^{\mu}}: \mathrm{e}^{-i q_{\mu}^{+} X_{+}^{\mu}\left(z_{+}\right)-i q_{\mu}^{-} X_{-}^{\mu}\left(z_{-}\right)}:$are the left-right symmetric tachyon vertex operators which form a basis for $\mathcal{A}_{X}$. Here $\Omega=\left\{\left(r^{(+) i}, r^{(-) i}\right) ; n_{j}, m_{k}\right\}$ labels the fields and ":" denotes normal ordering. The action of the operators (2) on the unique vacuum state $|\mathrm{vac}\rangle=1 \otimes|0\rangle_{+} \otimes|0\rangle_{-}$of $\mathcal{H}_{X}$ establishes the operator-state correspondence, implying that $\mathcal{A}_{X} \mathcal{H}_{X}=\mathcal{H}_{X}$. For a typical state $\left|\varphi_{q^{+} q^{-}}^{\Omega}\right\rangle=\left|q^{+} ; q^{-}\right\rangle \otimes \prod_{j} r_{\mu}^{(+) j} \alpha_{-n_{j}}^{(+)}|0\rangle_{+} \otimes$ $\prod_{k} r_{\nu}^{(-) k} \alpha_{-m_{k}}^{(-) \nu}|0\rangle_{-}$in $\mathcal{H}_{X}$, we have $\left|\varphi_{q^{+} q^{-}}^{\Omega}\right\rangle=V_{\Omega}\left(q^{+}, q^{-}\right) \mid$vac $\rangle$. The operator (2) is said to create a string state of type $\Omega$ and momentum $\left(q^{+}, q^{-}\right) \in \Gamma \oplus \Gamma^{*}$. The coefficients of the monomials in $z_{ \pm}$that arise in expansions of products of the tachyon vertex operators span the linear space generated by the operators (3) as the momenta $\left(q^{+}, q^{-}\right)$are varied. The noncommutativity of the algebra $\mathcal{A}_{X}$ is expressed in terms of the operator product expansion formula of the vertex operators, which in turn encodes the complete set of relations of the quantum field algebra and of $\mathcal{A}_{X}$ [7]. The vertex operators (3) generate all string scattering amplitudes, and so the noncommutative algebra $\mathcal{A}_{X}$ represents the "space" of interacting strings.

Finally, we introduce a Dirac operator. The chiral-antichiral decomposition in (1) allows two such operators to be introduced. The generators of target space reparametrizations $X_{ \pm}^{\mu}\left(z_{ \pm}\right) \rightarrow X_{ \pm}^{\mu}\left(z_{ \pm}\right)+\delta X_{ \pm}^{\mu}\left(z_{ \pm}\right)$, with $\delta X_{ \pm}^{\mu}\left(z_{ \pm}\right)$arbitrary periodic functions, are the conserved currents $\delta_{ \pm}^{\mu}\left(z_{ \pm}\right)=-i z_{ \pm} \partial_{z_{ \pm}} X_{ \pm}^{\mu}\left(z_{ \pm}\right)$in each chiral sector. To define the Dirac operator, we need to augment the Hilbert space $\mathcal{H}_{X}$ to incorporate spinors. Thus we consider instead $\mathcal{H}=L^{2}\left(\operatorname{spin}\left(T^{d}\right)\right)^{\Gamma} \otimes \mathcal{F}^{+} \otimes \mathcal{F}^{-}$. Locally, the spin bundle splits into two chiral parts, so that the $L^{2}$-spinors split into left- and right-moving components via the 
decomposition $L^{2}\left(\operatorname{spin}\left(T^{d}\right)\right)=\mathcal{S}\left(T^{d}\right)^{+} \otimes \mathcal{S}\left(T^{d}\right)^{-} \otimes L^{2}\left(T^{d}\right)$, with $\mathcal{S}\left(T^{d}\right)^{ \pm}$two anticommuting representations of the corresponding Clifford algebra with gamma-matrices $\gamma_{\mu}^{ \pm}$. The $C^{*}$-algebra $\mathcal{A}=\mathbb{I} \otimes \mathcal{A}_{X}$ acts trivially on the spinor part of $\mathcal{H}$. We then define two mutually anticommuting Dirac operators by

$$
D^{ \pm}\left(z_{ \pm}\right)=\sqrt{2} \gamma_{\mu}^{ \pm} \delta_{ \pm}^{\mu}\left(z_{ \pm}\right)=\sum_{k=-\infty}^{\infty} \sqrt{2} \gamma_{\mu}^{ \pm} \alpha_{k}^{( \pm) \mu} z_{ \pm}^{-k}
$$

with $\alpha_{0}^{( \pm) \mu}=g^{\mu \nu} p_{\nu}^{ \pm}$. The Dirac operators (4i) are related [7] to Witten's Dirac-Ramond operator associated with $N=1$ superstrings [8]. These Dirac operators have been used recently by Chamseddine in [9] to construct an effective superstring action using the spectral action principle of noncommutative geometry [10].

The two natural choices of Dirac operator in the spectral triple $\mathcal{T}$ are thus $D=$ $\frac{1}{\sqrt{2}}\left(D^{+}+D^{-}\right)$and $\bar{D}=\frac{1}{\sqrt{2}}\left(D^{+}-D^{-}\right)$. The existence of both $D$ and $\bar{D}$ severely restricts the effective spacetime geometry and is intimately related to the fact that the quantum spacetime determined by the string theory is not $T^{d}$, but rather its quotient under the action of the duality group $G$ of the string theory. The point is that there exist several linear transformations $T: \mathcal{H} \rightarrow \mathcal{H}$ with $\bar{D}=T D T^{-1}$ which define automorphisms of the smeared vertex operator algebra, i.e. $T \mathcal{A} T^{-1}=\mathcal{A}$. Given that the change $T$ in Dirac operator simply corresponds to a change of metric on the spacetime, general covariance implies that the two spacetimes represented by the corresponding spectral triples are equivalent,

$$
(\mathcal{H}, \mathcal{A}, D) \cong(\mathcal{H}, \mathcal{A}, \bar{D})
$$

More complicated duality symmetries can also be constructed by introducing a larger set of Dirac operators associated with, say, an $N=2$ supersymmetric sigma-model [1] which contains a larger symmetry than just the chiral-antichiral one exploited above.

The spacetime duality maps $T$ are, by definition, those which preserve the low-energy spectral triple $\mathcal{T}_{0}$. They are determined by the various mappings between the subspaces

$$
\mathcal{H}_{0} \equiv \operatorname{ker} D=\bigotimes_{\mu=1}^{d}\left(\mathcal{H}_{0}^{(+) \mu} \oplus \mathcal{H}_{0}^{(-) \mu}\right)
$$

and those of the analogously constructed $\overline{\mathcal{H}}_{0}=\operatorname{ker} \bar{D}$. The kernel (6) is composed of $2^{d}$ subspaces each of which represents a particular choice of spin structure on the $d$-torus, i.e. a choice of antiperiodic or periodic fermionic boundary conditions around each of the $d$ circles of $T^{d}$. In $\mathcal{H}_{0}^{(+) \mu}\left(\right.$ resp. $\left.\mathcal{H}_{0}^{(-) \mu}\right)$, the states satisfy $g^{\nu \lambda} d_{\lambda \mu}^{+} \gamma_{\nu}^{+}=g^{\nu \lambda} d_{\lambda \mu}^{-} \gamma_{\nu}^{-}\left(\gamma_{\mu}^{+}=-\gamma_{\mu}^{-}\right)$ and $p_{\mu}=0\left(w^{\mu}=0\right)$. On the other hand, in $\overline{\mathcal{H}}_{0}^{(+) \mu}$ (resp. $\left.\overline{\mathcal{H}}_{0}^{(-) \mu}\right)$, we have $\gamma_{\mu}^{+}=\gamma_{\mu}^{-}$ $\left(g^{\nu \lambda} d_{\lambda \mu}^{+} \gamma_{\nu}^{+}=-g^{\nu \lambda} d_{\lambda \mu}^{-} \gamma_{\nu}^{-}\right)$and $w^{\mu}=0\left(p_{\mu}=0\right)$. In all of these subspaces we also have $\alpha_{k}^{( \pm) \nu}=0$ for all $\nu$ and all $k>0$. This is precisely what is meant by the low-energy sector of the string theory, the internal motion of the string is suppressed leaving only its particle-like center of mass degrees of freedom. 
We immediately find an explicit isomorphism $\mathcal{H}_{0}^{(+) \mu} \leftrightarrow \mathcal{H}_{0}^{(-) \mu}$ defined by $g^{\nu \lambda} d_{\lambda \mu}^{ \pm} \gamma_{\nu}^{ \pm} \leftrightarrow$ $\pm \gamma_{\mu}^{ \pm}$and $g^{\mu \nu} p_{\nu} \leftrightarrow w^{\mu}$, and similarly on $\overline{\mathcal{H}}_{0}^{(+) \mu} \leftrightarrow \overline{\mathcal{H}}_{0}^{(-) \mu}$. This means that the low-energy effective spacetime is independent of the choice of spin structure on $\left(S^{1}\right)^{d}$. Thus a change of spin structure manifests itself as a T-duality symmetry of the string theory. We can therefore make the canonical choice of antichiral low-energy subspace $\mathcal{H}_{0}^{(-)}=\mathcal{H}_{0}^{(-) 1} \otimes \cdots \otimes$ $\mathcal{H}_{0}^{(-) d}$ determined by $D$. In this subspace, the states carry the antichiral representation $\gamma_{\mu}^{+}=-\gamma_{\mu}^{-} \equiv \gamma_{\mu}$ for all $\mu$, and they have $w^{\mu}=0$ for all $\mu$ so that $p_{\mu}^{+}=p_{\mu}^{-}=p_{\mu} / \sqrt{2} \in \Gamma^{*}$, as is required since in the low-energy sector there should be no non-local string modes which wind around the compactified directions. It is straightforward to show that $\mathcal{H}_{0}^{(-)}$ is naturally isomorphic to the Hilbert space $L^{2}\left(\operatorname{spin}\left(T^{d}\right)^{-}\right)$of antichiral square-integrable spinors on the torus, and that the action of the antichiral Dirac operator $D^{-}$on $\mathcal{H}_{0}^{(-)}$is $\left.D^{-}\right|_{\mathcal{H}_{0}^{(-)}}=i g^{\mu \nu} \gamma_{\mu} \frac{\partial}{\partial x^{\nu}}$.

It remains to construct a low-energy projection $\mathcal{A}_{0}$ of $\mathcal{A}$. We define $\mathcal{A}_{0}$ to be the commutant of $D$,

$$
\mathcal{A}_{0}=\operatorname{comm} D=\{V \in \mathcal{A} \mid[D, V]=0\}
$$

It is the largest subalgebra of $\mathcal{A}$ with the property $\mathcal{A}_{0} \mathcal{H}_{0}=\mathcal{H}_{0}$. It can be shown that $\left.\mathcal{A}_{0}\right|_{\mathcal{H}_{0}^{(-)}}$consists precisely of those vertex operators $V_{\Omega}\left(q^{+}, q^{-}\right)$which create string states of identical left and right chiral momentum, $q_{\mu}^{+}=q_{\mu}^{-}=q_{\mu} / \sqrt{2} \in \Gamma^{*}$. Furthermore, the smeared tachyon generators $V(q, q)$ of $\left.\mathcal{A}_{0}\right|_{\mathcal{H}_{0}^{(-)}}$coincide with the spacetime functions $\mathrm{e}^{-i q_{\mu} x^{\mu}}$ which constitute a basis for the algebra $C^{0}\left(T^{d}\right)$. Thus, the antichiral low-energy projection of the left-hand side of (5) is

$$
\begin{aligned}
\mathcal{T}_{0}^{(-)} & =\left(\mathcal{H}_{0}^{(-)},\left.\mathcal{A}_{0}\right|_{\mathcal{H}_{0}^{(-)}},\left.D^{-}\right|_{\mathcal{H}_{0}^{(-)}}\right) \\
& =\left(L^{2}\left(\operatorname{spin}\left(T^{d}\right)^{-}\right), C^{0}\left(T^{d}\right), i g^{\mu \nu} \gamma_{\mu} \partial_{\nu}\right)
\end{aligned}
$$

which describes the toroidal spacetime $T^{d}$ with metric $g_{\mu \nu}$.

Now let us carry out the same construction for $\bar{D}$, choosing again the corresponding antichiral low-energy subspace $\overline{\mathcal{H}}_{0}^{(-)}$. In this subspace the states carry the antichiral spinor representation $g^{\nu \lambda} d_{\lambda \mu}^{+} \gamma_{\nu}^{+}=-g^{\nu \lambda} d_{\lambda \mu}^{-} \gamma_{\nu}^{-} \equiv \tilde{\gamma}_{\mu}$ and have $p_{\mu}=0$ for all $\mu$, so that $\left(d^{+}\right)^{\mu \nu} p_{\nu}^{+}=-\left(d^{-}\right)^{\mu \nu} p_{\nu}^{-}=w^{\mu} / \sqrt{2} \in \Gamma$. These conditions are T-dual to those of $\mathcal{H}_{0}^{(-)}$. In the present context, T-duality is the linear isomorphism $T$ in (5) that maps $T\left|p^{+} ; p^{-}\right\rangle=(-1)^{p_{\mu} w^{\mu}}\left|\left(d^{+}\right)^{-1} p^{+} ;-\left(d^{-}\right)^{-1} p^{-}\right\rangle, T \alpha_{k}^{( \pm) \mu} T^{-1}= \pm g_{\nu \lambda}\left(d^{\mp}\right)^{\mu \nu} \alpha_{k}^{( \pm) \lambda}$, and $T \gamma_{\mu}^{ \pm} T^{-1}=g^{\nu \lambda} d_{\mu \nu}^{\mp} \gamma_{\lambda}^{ \pm}$. This implies that the target space metric changes to its dual $\tilde{g}^{\mu \nu}=\left(d^{+}\right)^{\mu \lambda} g_{\lambda \rho}\left(d^{-}\right)^{\rho \nu}$ which defines a metric on $\Gamma^{*} . T$ acts on the smeared vertex operator algebra as $T V_{\Omega}\left(q^{+}, q^{-}\right) T^{-1}=(-1)^{q_{\mu} w^{\mu}} V_{\Omega}\left(q^{+}\left(d^{+}\right)^{-1},-q^{-}\left(d^{-}\right)^{-1}\right)$, and so this linear mapping preserves both $\mathcal{H}$ and $\mathcal{A}$ at the same time as establishing the spectrum-preserving mapping $\bar{D}=T D T^{-1}$. This transformation is the noncommutative geometry version of the celebrated T-duality transformation of string theory which exchanges the torus $T^{d}$ with its dual $\left(T^{d}\right)^{*} \cong \mathbb{R}^{d} / 2 \pi \Gamma^{*}$, and interchanges momenta and windings in the spectrum. It corresponds to an inversion of the background matrices $d^{ \pm} \rightarrow\left(d^{ \pm}\right)^{-1}$ and is the 
$d$-dimensional analog of the $R \rightarrow 1 / R$ circle duality [1].

In the low-energy sector, it is once again possible to show that $\overline{\mathcal{H}}_{0}^{(-)}=L^{2}\left(\operatorname{spin}^{*}\left(T^{d}\right)^{-}\right)$, that $\left.D^{-}\right|_{\overline{\mathcal{H}}_{0}^{(-)}}=i \tilde{g}^{\mu \nu} \tilde{\gamma}_{\mu} \frac{\partial}{\partial x^{\nu}}$ and $\left.\overline{\mathcal{A}}_{0}\right|_{\overline{\mathcal{H}}_{0}^{(-)}}=\left.\operatorname{comm} \bar{D}\right|_{\overline{\mathcal{H}}_{0}^{(-)}}=C^{0}\left(T^{d}\right)$, yielding the $\mathrm{T}$-dual low-energy triple $\overline{\mathcal{T}}_{0}^{(-)}$to (8). Then according to (5), as subspaces of the effective quantum spacetime we have the equivalence between $\left(L^{2}\left(\operatorname{spin}\left(T^{d}\right)^{-}\right), C^{\infty}\left(T^{d}\right), i g^{\mu \nu} \gamma_{\mu} \partial_{\nu}\right)$ and $\left(L^{2}\left(\operatorname{spin}^{*}\left(T^{d}\right)^{-}\right), C^{\infty}\left(T^{d}\right), i \tilde{g}^{\mu \nu} \tilde{\gamma}_{\mu} \partial_{\nu}\right)$ which is the usual statement of the T-duality symmetry $T^{d} \leftrightarrow\left(T^{d}\right)^{*}$ of bosonic string theory compactified on a $d$-torus. Notice how the statement that this duality symmetry corresponds to the target space symmetry $g_{\mu \nu} \leftrightarrow \tilde{g}^{\mu \nu}$ and the symmetry under interchange of momentum and winding in the compactified string spectrum arise very naturally from the point of view of noncommutative geometry. The crucial aspect of the low-energy projections is that the restricted vertex operator subalgebras consist of only the zero mode components of the tachyon vertex operators in (3).

There are many other symmetries that arise from such isomorphisms, corresponding to mappings from $\mathcal{H}_{0}^{(-)}$onto other subspaces of ker $\bar{D}$. For example, worldsheet parity and factorized duality (equivalently mirror symmetry when $d$ is even) easily arise in this way [7]. Besides these geometrical symmetries, there are two other internal symmetries of the spacetime which trivially leave the spectral triples in (5) invariant. The first is a change of basis of the lattice $\Gamma$, a process which preserves all quantities composing the spectral triples. The second is the global shift $\beta_{\mu \nu} \rightarrow \beta_{\mu \nu}+C_{\mu \nu}$ of the instanton tensor by an antisymmetric integer-valued matrix $C_{\mu \nu}$, which can be absorbed in a shift of the momenta $p_{\mu} \rightarrow p_{\mu}-C_{\mu \nu} w^{\nu}$ and leaves the spectral triples unaffected. It can be shown that the set of duality transformations described here generate the discrete duality group of the string theory which is the semidirect product $G=O(d, d ; \mathbb{Z}) \otimes_{\mathrm{S}} \mathbb{Z}_{2}$ of the lattice automorphism group $O(d, d ; \mathbb{Z})$ and the reflection group $\mathbb{Z}_{2}$ corresponding to worldsheet parity. The above constructions thus show how the geometry and topology relevant for general relativity must be embedded in a larger noncommutative structure in string theory. They can also be generalized to include worldsheet gravitational effects and more complicated target space geometries [7], as well as to obtain duality maps between different spectral triples representing the equivalences of distinct string theories and D-brane models as dictated by $\mathrm{M}$ Theory [2]. The duality group is a subgroup of the full group of automorphisms of the algebra $\mathcal{A}$ which represents the diffeomorphism and internal symmetries of the stringy general relativity. In fact, $G$ appears in terms of the zero modes of the Dirac operators from (6), while the full set of automorphisms of the spectral triple $\mathcal{T}$ are determined by mappings between different Dirac operators that have the same spectrum [10]. Thus the full set of symmetries of the string spacetime is intimately connected with some of the exotic mathematical structures which appear in the theory of vertex operator algebras, such as the Monster Lie algebra [11]. More details about these constructions will appear in a forthcoming paper [7]. 


\section{References}

[1] A. Giveon, M. Porrati and E. Rabinovici, Phys. Rep. 244 (1994) 77.

[2] E. Witten, Nucl. Phys. B443 (1995) 85; J.H. Schwarz, Phys. Lett. B367 (1996) 97; Nucl. Phys. B (Proc. Suppl.) 55B (1997) 1; M.J. Duff, Int. J. Mod. Phys. A11 (1996) 5623.

[3] E. Witten, Nucl. Phys. B460 (1996) 335.

[4] A. Connes, Noncommutative Geometry (Academic Press, 1994); G. Landi, An Introduction to Noncommutative Spaces and their Geometry, to appear as Springer Lecture Notes in Physics, hep-th/9701078.

[5] M.R. Douglas, in Quantum Symmetries, Proc. LXIV Les Houches Session, eds. A. Connes and K. Gawędzki, to appear, hep-th/9610041; P.-M. Ho and Y.-S. Wu, Phys. Lett. B398 (1997) 52; J. Kalkkinen, ibid. B399 (1997) 243.

[6] J. Fröhlich and K. Gawędzki, CPM Proc. Lecture Notes 7 (1994) 57; see also A.H. Chamseddine and J. Fröhlich, in Yang Festschrift, eds. C.S. Lui and S.-F. Yau (International Press, Boston, 1995).

[7] F. Lizzi and R.J. Szabo, in preparation.

[8] E. Witten, Nucl. Phys. B202 (1982) 253; ibid., B268 (1986) 253.

[9] A.H. Chamseddine, Phys. Lett. B400 (1997) 87; hep-th/9705153.

[10] A.H. Chamseddine and A. Connes, Phys. Rev. Lett. 77 (1996) 4868; to appear in Commun. Math. Phys., hep-th/9606001.

[11] I.B. Frenkel, J. Lepowsky and A. Meurman, Vertex Operator Algebras and the Monster (Academic, New York, 1988). 